\title{
A Case of Eosinophilic Gastrointestinal Disorders Presenting with Chronic Diarrhea and Abdominal Pain

\author{
Dong-Ryul Lee*
}

Department of Family Medicine, Sanbon Medical Center, Wonkwang University College of Medicine, Gunpo, Korea

Eosinophilic gastrointestinal disorders (EGID) are rare conditions characterized by eosinophilic infiltration of the bowel wall and gastrointestinal symptoms. These disorders can present with various manifestations, depending on the specific site of the affected gastrointestinal tract and specific layer of the affected gastrointestinal wall. The pathogenesis and etiology of EGID remain unclear. The present case report describes a 76-year-old woman who presented with chronic diarrhea and lower abdominal pain. The patient was successfully treated with corticosteroid and restriction of some food allergens. One needs to consider these rare disorders during the differential diagnosis of unexplained gastrointestinal symptoms, especially when they are associated with peripheral eosinophilia.

Keywords: Eosinophilic Gastrointestinal Disorders; Eosinophilia; Chronic Diarrhea

\section{INTRODUCTION}

Chronic diarrhea is a common condition in primary care. ${ }^{1)}$ The various definitions of chronic diarrhea commonly include the presence of loose stools that last for at least 4 weeks. By one estimate, it occurs in up to $3-5 \%$ of population in United States. Pathophysiologically, the major causes of chronic diarrhea include osmotic, secretory, inflammatory, and functional factors. Chronic diarrhea is a treatment challenge, even for experienced clinicians. ${ }^{1)}$

Received: December 29, 2010, Accepted: May 19, 2011

*Corresponding Author: Dong-Ryul Lee

Tel: 82-31-390-2965, Fax: 82-31-390-2746

E-mail: rednose1@hanmail.net

Korean Journal of Family Medicine

Copyright $(92011$ by The Korean Academy of Family Medicine

() This is an open-access article distributed under the terms of the Creative Commons Attribution Non-Commercial License (http://creativecommons.org/licenses/by-nc/3.0) which permits unrestricted noncommercial use, distribution, and reproduction in any medium, provided the original work is properly cited.
Therefore, family medicine doctors as primary physicians should be aware of systematic approaches and various experiences pertaining to this condition.

Eosinophilic gastrointestinal disorders (EGID) are one of rare causes of chronic diarrhea. The disorders are characterized by inflammation rich in eosinophilic infiltration in the gastrointestinal (GI) tract without evidence of known causes for eosinophilia such as parasitic infection, drug reaction, or malignancy. ${ }^{3-5)}$ It was originally described by Kaijser ${ }^{6)}$ in 1937; since then, hundreds of cases have been described from all over the world including North America, Europe, Australia, and Asia. ${ }^{7)}$ Diverse cases of EGID have also been reported in Korea. ${ }^{8,9)}$ EGID can involve one or multiple segments of the GI tract from the esophagus to the rectum (mainly in the antrum of the stomach and small intestine), ${ }^{10)}$ and can also occupy various sites through the depth of the wall. Such inconsistency from case to case promotes unpredictable symptoms from pain to dysmotility, bleeding, obstruction, or ascites. ${ }^{11)}$

About half of EGID cases present with allergic features, and some may be related to food allergy. ${ }^{12)}$ However, there are few reports of EGID associated with adults, especially with the 
elderly. ${ }^{13,14)}$ The present case report describes an elderly woman who presented with chronic diarrhea with lower abdominal pain. Marked improvement resulted from steroid therapy and restriction of some food allergens.

\section{CASE REPORT}

A 76-year-old woman visited our health promotion center for a health check-up with diarrhea, abdominal pain, and weight loss. About 2 years before, the patient had developed intermittent watery diarrhea occurring after meals. Five months previous to the check-up, the diarrhea had become aggravated, with an average of 9 or 10 stool passages each day. Stool was yellowish and watery in character, and ended with bloody mucus with poignant lower abdominal pain. During this period, body weight decreased from $48 \mathrm{~kg}$ to $35 \mathrm{~kg}$, and was accompanied by generalized weakness. The patient had been admitted to other hospitals several times and had received a medical check-up including a colonoscopy. However, no specific cause of the chronic diarrhea had been identified. One week previous to the current checkup, the symptoms had recurred, which prompted the visit to our center.

The patient had no personal and family history of allergic disorders such as asthma, atopy, allergic rhinitis, and other hypersensitivities, and denied any exposure to tobacco smoke, alcohol, drugs, herbal medications, and health functional foods. Laboratory findings were as follows: leukocyte count $8,800 / \mathrm{mm}^{3}$ (neutrophil, 40.5\%; lymphocyte, 39.7\%; eosinophil, 13.4\%), total eosinophil count $1,180 / \mathrm{mm}^{3}$ (normal range, 0 to $500 /$ $\mathrm{mm}^{3}$ ), hemoglobin $9.7 \mathrm{~g} / \mathrm{dL}$ (mean corpuscular volume, 95.3 $\mathrm{fl})$, erythrocyte sedimentation rate $21 \mathrm{~mm} /$ hour, and C-reactive protein $0.233 \mathrm{mg} / \mathrm{dL}$. Biochemical tests were within normal limits other than protein $(5.49 \mathrm{~g} / \mathrm{dL}$; normal range, 6.7 to $8.3 \mathrm{~g} /$ $\mathrm{dL})$ and albumin $(2.74 \mathrm{~g} / \mathrm{dL}$; normal range, 3.8 to $5.0 \mathrm{~g} / \mathrm{dL})$. Stool was positive for occult blood and negative for ova and parasites. Upper GI endoscopy revealed mild antral gastritis. But, colonoscopy showed multifocal hyperemic edematous
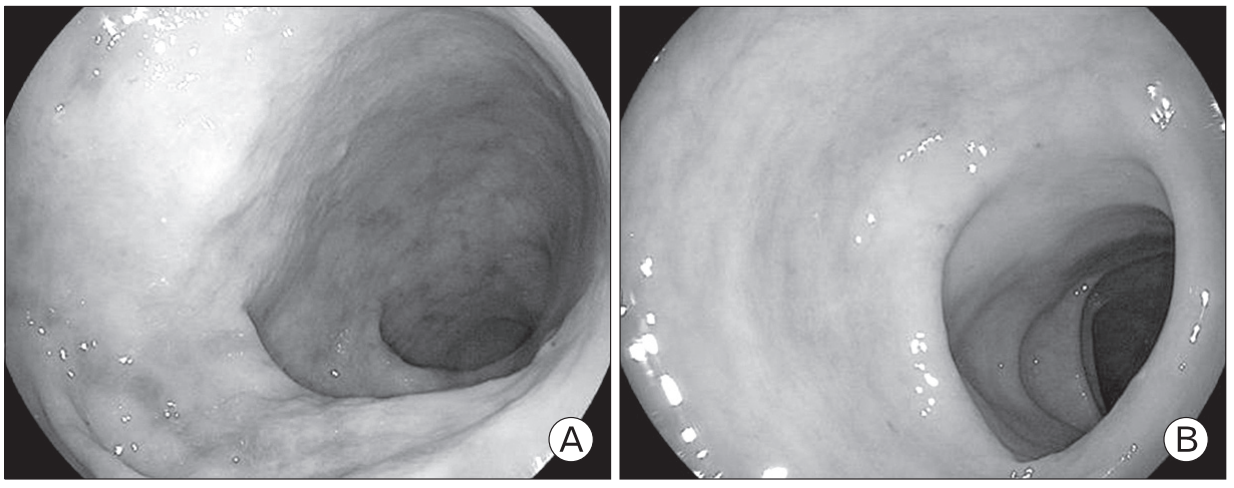

Figure 1. Colonoscopic findings. (A) Hyperemic edematous mucosal change was evident on the descending colon. (B) Mild edematous mucosal change was noted on the sigmoid colon.
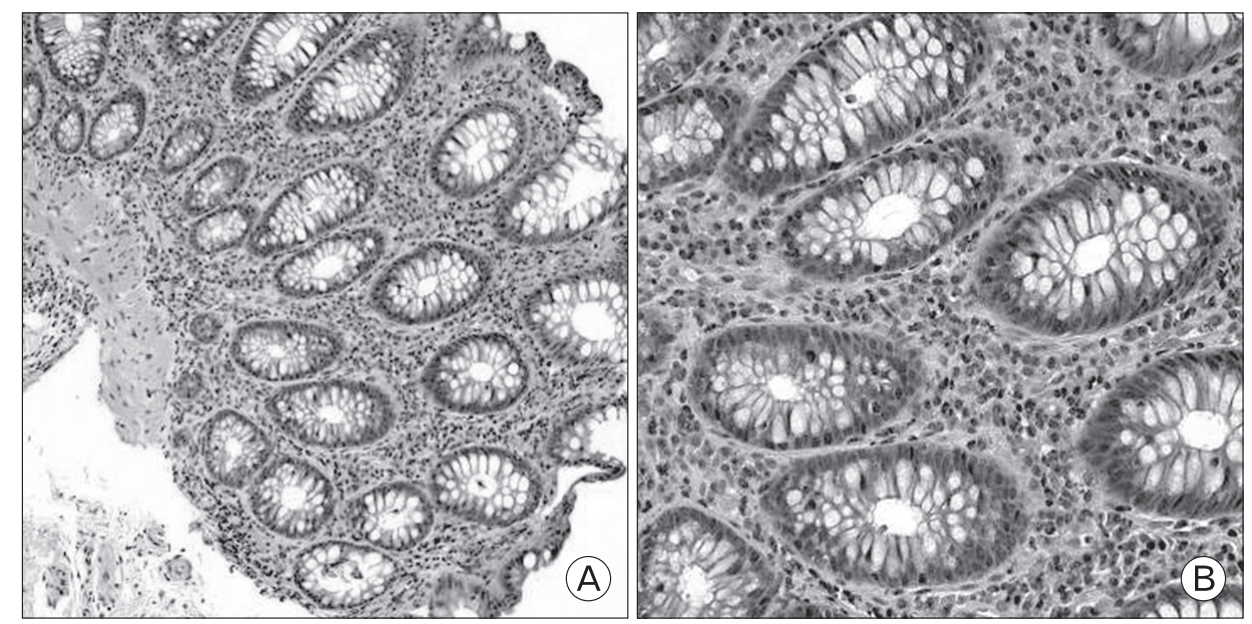

Figure 2. Pathologic findings. Endoscopic biopsy showing diffuse infiltration of eosinophils and lymphocytes in the lamina propria on the descending colon. There are more than 20 eosinophils per high power field (A, $H \& E \times 200$; B, H\&E, $\times 400$ ). 
mucosa from the cecum to the sigmoid colon (Figure 1). Multiple random biopsies were taken. The pathologic findings revealed diffuse infiltration of eosinophils and lymphocytes in the lamina propria, especially on the descending colon (Figure 2). Abdominal computed tomography was unremarkable except for a simple renal cyst.

The patient was admitted to our hospital for further evaluation. On admission, the patient appeared chronically ill. Vital signs were stable including blood pressure $(110 / 70 \mathrm{~mm} \mathrm{Hg})$, heart rate (74/minute), respiration rate (20/minute), and body temperature $\left(36.5^{\circ} \mathrm{C}\right)$. The conjunctiva was anemic and the sclera was anicteric. There was no cervical lymphadenopathy. On auscultation, the lung fields were clear. Cardiac examination revealed no murmur or gallop. Abdominal examination revealed hyperactive bowel sound and mild tenderness below the umbilicus. Extremities were not edematous or cyanotic. Neurological examination revealed no deficits or muscle weakness.

Results of additional stool exam for leukocytes and fat were normal and stool culture was negative. Laboratory examination for anemia revealed ferritin $27.59 \mathrm{ng} / \mathrm{mL}$, serum iron $26.39 \mathrm{ng} /$ $\mathrm{dL}$, total iron-binding capacity $156.39 \mathrm{ng} / \mathrm{dL}$, red cell distribution width $15.6 \%$, and reticulocyte index 0.357 . The results were consistent with iron deficiency anemia coexisting with anemia of chronic disease.

As an additional evaluation for eosinophilia, tests for parasite specific antigens to Clonorchis, Paragonimus, Sparganum, and Cysticercus were negative. Serological tests for connective tissue disease and vasculitis (rheumatoid factor, antinuclear antibody, lupus erythematosus test, and anti-neutrophil cytoplasmic antibody) were also negative. Total immunoglobulin E ( $\operatorname{IgE}$ ) was $46.63 \mathrm{IU} / \mathrm{mL}$ (normal range, 0 to $120 \mathrm{IU} / \mathrm{mL}$ ) and multiple allergosorbent test (MAST) test for 35 allergens including soy, milk, wheat, and house dust mite was negative for all allergens.

After admission, the patient's condition was improved by supportive care such as soft blend diet and parenteral hydration. However, symptoms recurred soon after discharge. Based on the symptoms of chronic diarrhea with lower abdominal pain, peripheral eosinophilia and eosinophilic infiltration on colonoscopic biopsy without any other causes, a diagnosis of EGID (subtype, eosinophilic colitis) was made. The patient received steroid therapy (prednisolone, $20 \mathrm{mg} /$ day). Three days later, symptoms had markedly improved. One week later, the peripheral eosinophilia had resolved $\left(239 / \mathrm{mm}^{3}\right)$. After 2 weeks of treatment, steroid was tapered over a week. However, symptoms recurred, and cromolyn sodium and montelukast were tried to no avail.

According to the report that EGID are associated with food allergy, ${ }^{12)}$ the patient was instructed to maintain a food diary. Wheat, soy, and milk were suspected as allergens; restriction of these items from the diet produced a greater improvement in the absence of steroid use. The patient has not had a clear relapse for 5 months after discharge and has gained $7 \mathrm{~kg}$ in weight. Currently, there is no limitation in activities of daily living, and the patient takes only low-dose of prednisolone $(5 \mathrm{mg} /$ day $)$ intermittently for episodic mild diarrhea.

\section{DISCUSSION}

EGID are rare conditions characterized by eosinophilic infiltration of the bowel wall and gastrointestinal symptoms. ${ }^{3,4)}$ In 1990, Talley et al. ${ }^{15)}$ defined EGID by three criteria: 1 ) the presence of gastrointestinal symptoms, 2) biopsies showing eosinophilic infiltration of one or more areas of the gastrointestinal tract from the esophagus to the colon, or characteristic radiological findings with peripheral eosinophilia, and 3) no evidence of parasitic or extraintestinal disease. Presently, this concept is most commonly used in defining EGID.

In published reports, stomach $(26-81 \%)$ and small intestine $(28-100 \%)$ are the predominantly affected sites but esophagus, large intestine, and rectum may be affected as well. ${ }^{7)}$

EGID consist of heterogeneous subtypes including eosinophilic esophagitis, eosinophilic gastritis, eosinophilic gastroenteritis, and eosinophilic colitis. Although some researchers regard eosinophilic gastroenteritis as a synonym for EGID, others have used this term to refer to eosinophilic inflammation exclusively to the stomach and small intestine. To avoid redundancy in this issue, this article restricted eosinophilic gastroenteritis as the latter. ${ }^{16)}$

EGID are exceedingly rare, lacking epidemiological data to estimate their true frequency. EGID affect all races and ages, from infancy through adulthood, peaking during the second and sixth decades of life. ${ }^{5)}$ Contrary to other subtypes, eosinophilic colitis 
has a bimodal age distribution, usually affecting infants (mean age, 60 days) and adolescents. ${ }^{17)}$ Although there are some differences among the reports, the incidence is higher in males, with a ratio of $3: 2$. $^{5)}$

The etiology of EGID remains largely unknown. Several studies have suggested a relationship with specific food allergies, and approximately $70 \%$ of patients may have a personal or family history of allergic disorders such as asthma, hay fever, hypersensitivity to drugs, or eczema. ${ }^{5)}$ Thus, EGID may be associated with hypersensitivity reactions. Recent investigations have provided an insight into the pathogenesis of EGID, and support a critical role of allergens, eosinophils, mast cells, Th-2 type cytokines (interleukin-3, -5, and -13), and eotaxin in mediating eosinophilic inflammation. ${ }^{18)}$

Although EGID are supposed to be related with hypersensitivity, immunologic evidence of underlying allergy is lacking in most cases, except those mediated by $\operatorname{IgE}$ antibody, and typically EGID are present as immediate reactions or accompanied by atopy or asthma. In contrast to other EGID, eosinophilic colitis is usually a non-IgE-associated disease. Thus, skin prick test or antigen-specific IgE (RAST) is negative. ${ }^{19)}$ Some studies point to a $\mathrm{T}$ cell-mediated process, but the exact immunologic mechanisms for this have not been identified. ${ }^{3,13)}$ Similarly, the present case was normal in total and antigen-specific IgE (MAST) and lacked immunologic evidence for allergy. But, dramatic improvement noted upon restriction of some foods provides strong evidence of a hypersensitivity reaction.

Signs and symptoms of EGID vary depending upon the location and layer of the involved gastrointestinal tissue. Classification of EGID by layer of GI involvement was proposed by Klein et al. ${ }^{10)}$ in 1970 , and is currently the most referred in publication. Mucosal involvement is by far the most common, and is accompanied by one or more of the following symptoms: decreased appetite, nausea, vomiting, abdominal pain, diarrhea, GI bleeding, protein-losing enteropathy, anemia, and failure to thrive. Involvement of the muscular layer usually presents with colicky abdominal pain and symptoms of gastric outlet or intestinal obstruction. Serosal involvement, the least common, is accompanied by abdominal distention and eosinophilic ascites. Combinations of these symptoms occur with the involvement of more than one tissue layer.

The diagnosis of EGID requires a high index of suspicion, as the symptoms and presentations are nonspecific, often sharing features with more commonly encountered GI disorders, such as infection, inflammatory bowel diseases, and iatrogenic and functional GI disorders. ${ }^{20)}$ There are no confirmatory laboratory tests. Peripheral blood eosinophilia is suggestive of EGID, but is noted in only $60-80 \%$ of the patients. ${ }^{21)}$ Laboratory tests are useful in distinguishing EGID from other diseases associated with tissue eosinophilia.

Endoscopic findings may be nonspecific and can include erythema, friability, ulcerations, erosions, nodules, and loss of vascularity. ${ }^{47}$ Because normal-appearing mucosa can harbor eosinophilic infiltration and because of the patchy nature of these disorders with the exception of eosinophilic esophagitis, the diagnosis can be overlooked or remains in question if multiple biopsies are not taken. When biopsy specimens are evaluated and eosinophilic infiltration is identified, other causes of eosinophil infiltration must be excluded before the diagnosis is confirmed. As a result, it is all very common for patients with these disorders to be symptomatic for extended periods before an accurate diagnosis is made. ${ }^{22)}$

Pathologic evaluation to prove eosinophilic infiltration is essential to confirm the diagnosis. Eosinophils are normally present in the lamina propria of the gut mucosa, except the esophagus. ${ }^{4)}$ There is generally no agreement on what constitutes a pathological number of eosinophils. In most of the publications involving large case series, a cut-off of $\geq 20$ eosinophils per high power field has been used to define EGID histologically. ${ }^{14,15,23)}$ In the present case, the tissue eosinophil numbers were also consistent with this definition. The diagnosis of the muscular form of EGID may require laparoscopic full-thickness biopsies. In eosinophilic colitis, there may be diffuse or focal aggregates in the lamina propria, crypt epithelium, and muscular mucosa preserving the underlying mucosal architecture. ${ }^{22)}$

The differential diagnosis of EGID includes secondary eosinophil-associated GI disorders, such as hypereosinophilic syndromes, parasitic infections, inflammatory bowel diseases, celiac disease, food allergies, neoplasia, connective tissue disorders (e.g., scleroderma and dermatomyositis) collagen vascular disorders (e.g., polyarteritis nodosa and Churg-Strauss syndrome), and use of medications. ${ }^{4,5,7)}$

Evaluation for idiopathic hypereosinophilic syndrome (HES) in patients with apparent EGID is important. ${ }^{3,7)}$ Diagnostic 
criteria for HES include 1) persistent eosinophilia of at least 1,500 cells $/ \mathrm{mm}^{2}$ for a minimum of 6 months, 2) lack of known causes for eosinophilia (e.g., parasitic or allergic triggers), and 3) symptoms and signs of organ system involvement. ${ }^{24)}$ Patients with EGID and blood eosinophil counts exceeding $1,500 / \mathrm{mm}^{2}$ meet these diagnostic criteria. However, patients with EGID are usually not at high-risk of life-threatening complications associated with classic idiopathic HES (i.e., cardiomyopathy or central nervous system involvement). ${ }^{3)}$

Treatment of EGID can be divided into dietary modifications and pharmacotherapy. The role of food allergy in EGID is not as well-defined as it is in eosinophilic esophagitis. Nevertheless, if specific food sensitivities can be identified, patients have responded to targeted elimination diets. In cases in which the identification of offending food antigens is arguable, an elemental diet might be helpful. ${ }^{24)}$ This strategy, which has been established with great success in children, is also used in adults, and has a very high response rate (close to $100 \%$ within 3 weeks), although it is difficult for most patients to maintain such a strict diet. ${ }^{12)}$

If no food sensitization is discovered, or if a targeted elimination diet is not feasible or has failed to alleviate symptoms, systemic (for acute exacerbations) or topical glucocorticoids can be considered for therapy. ${ }^{5,7)}$ The recommended dose is prednisolone $20-40 \mathrm{mg} /$ day for $1-2$ weeks. The dose is then tapered off over several weeks. ${ }^{4)} \mathrm{Up}$ to $90 \%$ of cases will respond dramatically within 2 weeks of treatment. ${ }^{22)}$ However, a maintenance does of prednisolone $(10 \mathrm{mg} /$ day $)$ and cessation of corticosteroids has resulted in recurrence of symptoms in $15 \%$ and $55 \%$ of patients, respectively. ${ }^{25)}$ In eosinophilic esophagitis, swallowing topical fluticasone did not produce significant differences in efficacy or relapse, compared to oral steroid, although fewer adverse events were noted for fluticasone. ${ }^{26)}$ Less well-documented treatments such as mast cell stabilizer (cromoglycate), leukotriene antagonist (montelukast), and antihistamines (ketotifen) have been reported in small, uncontrolled trials. ${ }^{25)}$ Experimental treatment with anti-IL-5 antibody (mepolizumab) seems, in general, to be the most promising, and is a putative treatment for severe or therapy-resistant EGID. ${ }^{27)}$ In severe cases, refractory or dependent on systemic glucocorticoid therapy, intravenous alimentation or immunosuppressive antimetabolite therapy (azathioprine or 6-mercaptopurine) are alternatives. $^{3)}$
Little is known about the natural history of EGID as it has not yet been evaluated in longitudinal clinical studies. However, from currently available data and recent experience with eosinophilic colitis, EGID are considered to be chronic waxing and waning disorders. ${ }^{22)}$ Several studies have suggested an association between allergic colitis and later development of inflammatory bowel disease (IBD), but this association is controversial. ${ }^{17)}$ Because these diseases can be predecessors to other disease processes, such as hypereosinophilic syndromes, routine endoscopic and cardiopulmonary evaluations are recommended. ${ }^{3)}$

In the present case, the patient presented with chronic diarrhea and lower abdominal pain and EGID (eosinophilic colitis) was documented by eosinophilic infiltration on endoscopic biopsy and exclusion of secondary causes. This patient was easily suspected of combined peripheral eosinophilia, but clinicians need to consider EGID as possible causes for chronic unexplained GI symptoms and take biopsies actively, although there are no characteristic endoscopic findings or peripheral eosinophilia.

\section{REFERENCES}

1. Schiller LR. Chronic diarrhea. Gastroenterology 2004;127: 287-93.

2. Fine KD, Schiller LR. AGA technical review on the evaluation and management of chronic diarrhea. Gastroenterology 1999;116:1464-86.

3. Rothenberg ME. Eosinophilic gastrointestinal disorders (EGID). J Allergy Clin Immunol 2004;113:11-28.

4. Yan BM, Shaffer EA. Primary eosinophilic disorders of the gastrointestinal tract. Gut 2009;58:721-32.

5. Khan S, Orenstein SR. Eosinophilic gastroenteritis: epidemiology, diagnosis and management. Paediatr Drugs 2002;4: 563-70.

6. Kaijser R. Zur Kenntnis der allergischen affektioner desima verdauungskanal standprnkt desima cirurgen aus. Arch Klin Chir 1937;188:36-64.

7. Feldman M, Scharschmidt B, Sleisenger M. Sleisenger and Fordtran's gastrointestinal and liver disease. 6th ed. Philadelphia: Saunders; 2006.

8. Park JS, Park BK, Kim KH. Idiopathic eosinophilic gastroenteritis in Korea: review of 90 collective cases. J Korean Surg 
Soc $1995 ; 48: 347-58$.

9. Kim NI, Jo YJ, Song MH, Kim SH, Kim TH, Park YS, et al. Clinical features of eosinophilic gastroenteritis. Korean J Gastroenterol 2004;44:217-23.

10. Klein NC, Hargrove RL, Sleisenger MH, Jeffries GH. Eosinophilic gastroenteritis. Medicine (Baltimore) 1970;49: 299-319.

11. Cello JP. Eosinophilic gastroenteritis: a complex disease entity. Am J Med 1979;67:1097-104.

12. Bischoff SC. Food allergy and eosinophilic gastroenteritis and colitis. Curr Opin Allergy Clin Immunol 2010;10:238-45.

13. Pratt CA, Demain JG, Rathkopf MM. Food allergy and eosinophilic gastrointestinal disorders: guiding our diagnosis and treatment. Curr Probl Pediatr Adolesc Health Care 2008;38:170-88.

14. Moneret-Vautrin AD. Gastrointestinal allergy in adults. Eur J Gastroenterol Hepatol 2005;17:1293-7.

15. Talley NJ, Shorter RG, Phillips SF, Zinsmeister AR. Eosinophilic gastroenteritis: a clinicopathological study of patients with disease of the mucosa, muscle layer, and subserosal tissues. Gut 1990;31:54-8.

16. Khan S, Orenstein SR. Eosinophilic gastroenteritis. Gastroenterol Clin North Am 2008;37:333-48.

17. Guajardo JR, Rothenberg ME. Eosinophilic esophagitis, gastroenteritis, gastroenterocolitis, and colitis. 3rd ed. Malden, MA: Blackwell Publishing; 2003.

18. Khan S. Eosinophilic gastroenteritis. Best Pract Res Clin Gastroenterol 2005; 19:177-98.

19. Conus S, Simon HU. General laboratory diagnostics of eosinophilic GI diseases. Best Pract Res Clin Gastroenterol
2008;22:441-53.

20. Straumann A. Idiopathic eosinophilic gastrointestinal diseases in adults. Best Pract Res Clin Gastroenterol 2008;22:481-96.

21. Straumann A, Simon HU. The physiological and pathophysiological roles of eosinophils in the gastrointestinal tract. Allergy 2004;59:15-25.

22. Fleischer DM, Atkins D. Evaluation of the patient with suspected eosinophilic gastrointestinal disease. Immunol Allergy Clin North Am 2009;29:53-63.

23. Whitington PF, Whitington GL. Eosinophilic gastroenteropathy in childhood. J Pediatr Gastroenterol Nutr 1988;7: 379-85.

24. Justinich C, Katz A, Gurbindo C, Lepage G, Chad Z, Bouthillier L, et al. Elemental diet improves steroid-dependent eosinophilic gastroenteritis and reverses growth failure. J Pediatr Gastroenterol Nutr 1996;23:81-5.

25. Ban GJ, Rho YH, Han S, Si S, Yoon SJ, Kim SY, et al. Diffuse eosinophilic gastroenteritis with tumor-like antral obstruction. Korean J Gastroenterol 1999;33:718-22.

26. Schaefer ET, Fitzgerald JF, Molleston JP, Croffie JM, Pfefferkorn MD, Corkins MR, et al. Comparison of oral prednisone and topical fluticasone in the treatment of eosinophilic esophagitis: a randomized trial in children. Clin Gastroenterol Hepatol 2008;6:165-73.

27. Straumann A, Conus S, Grzonka P, Kita H, Kephart G, Bussmann C, et al. Anti-interleukin-5 antibody treatment (mepolizumab) in active eosinophilic oesophagitis: a randomised, placebo-controlled, double-blind trial. Gut 2010; 59:21-30. 